\title{
Non-local Similarity Based Nonnegative Tucker Decomposition for Hyperspectral Image Denoising
}

\author{
Xiao Bai, Fan Xu, Lei Zhou, Yan Xing, Lu Bai, and Jun Zhou, Senior Member, IEEE
}

\begin{abstract}
Compared with color or grayscale images, hyperspectral images deliver more informative representation of ground objects and enhance the performance of many recognition and classification applications. However, hyperspectral images are normally corrupted by various types noises, which has serious impact on the subsequent image processing tasks. In this paper, we propose a novel hyperspectral image denoising method based on tucker decomposition to model the non-local similarity across the spatial domain and global similarity along the spectral domain. In this method, 3D full band patches extracted from a hyperspectral image are grouped to form a 3rd-order tensor by utilizing the non-local similarity in a proper window size. In this way the task of image denoising is transformed into a high order tensor approximation problem, which can be solved by nonnegative tucker decomposition. Instead of traditional alternative least square based tucker decomposition, we propose a hierarchical least square based nonnegative tucker decomposition method to reduce the computational cost and improve the denoising effect. In addition, an iterative denoising strategy is adopted to achieve better denoising outcome in practice. Experimental results on three datasets show that the proposed method outperforms several state-of-the-art methods and significantly enhances the quality of the corrupted hyperspectral image.
\end{abstract}

Index Terms-Hyperspectral Image, Denoising, Nonnegative Tucker Decomposition, Non-local Similarity, Hierarchical Alternative Least Square

\section{INTRODUCTION}

Hyperspectral image can be considered as a set of spatially organized "pixels" where each pixel contains an entire spectral reflectance at a spatial location in the image over given light wavelengths. Compared with grayscale or color images which integrate the spectral responses at only one or several light wavelength intervals, hyperspectral images deliver more detailed spectral information of real scenes. Numerous research have been undertaken on hyperspectral images, for example, in feature extraction [1], [2], unmixing [3]-[5], classification [6]-[10], detection [11]-[13], band selection [14] and surveillance [15]. However, conventional hyperspectral imaging process suffers from issues such as limited illumination and short sensing time which introduce noises into the image acquisition step. Therefore, denoising has become a critical step to improve the quality of hyperspectral images.

Fan $\mathrm{Xu}$, Lei Zhou and Xiao Bai are with the School of Computer Science and Engineering, Beihang University, Beijing 100191, China.

Yan Xing is with Beijing Institute of Control Engineering, Beijing 100080, China.

Lu Bai is with the School of Information, Central University of Finance and Economics, Beijing 102202, China.

Jun Zhou is with the School of Information and Communication Technology, Griffith University, Nathan, Australia.
Over the past a few years, several hyperspectral image denoising methods have been proposed. The most straightforward way is to utilize traditional 2D denoising methods, such as NLM [16], K-SVD [17] and BM3D [18], to reduce noise band by band separately. Such band-wised methods cannot achieve superior effect in many cases since only spatial noise is removed. They may also destroy the correlation of image data in the spatial or the spectral domain. Therefore, spatial and spectral information should be considered jointly to effectively remove the noises.

Consequently, a hyperspectral image can be treated as a whole entity rather than band by band processing. Several 3D patch based denoising methods have been put forward, such as adaptive non-local means (ANLM3D) [19], 3D cube K-SVD [20] and block matching 4D filtering (BM4D) [21]. 3D patch based denoising methods can be implemented by processing small 3D patches in both spatial space and spectral space. In many cases, only local spectral correlation between a few adjacent bands is considered, and the high spectral redundancy in all continuous spectral bands has not been fully used. Novel denoising methods [22]-[24] have been proposed recently by combining non-local similarity across the spatial space and global redundancy along the spectral space. However, when the noises are strong, image denoising methods based on sparse coding and low rank constraint perform poorly because the dictionary learning step tends to remove some inherent information, e.g. textures and edges [22].

To further exploit the spatial-spectral information to generate better noise free estimation, a hyperspectral image can be treated as a stack of 2D gray images, which can be seen as a 3rd-order tensor. Accordingly, tensor based dictionary learning method was explored [25], and some algorithms were proposed to approximate tensor based on tensor low rank approximation [26]-[28] or tensor decomposition [29]. In particular, low-rank approximation (LRTA) [30] utilizes low rank tensor approximation [31] of the input image to achieve denoising and spectral dimensionality reduction. PARAFAC [29] method further extends the denoising approach by employing parallel factor analysis. Though both approaches take the correlation over different bands into consideration and try to eliminate the spectral redundancy of hyperspectral image, they ignore the non-local similarity of full band patches across the spatial domain. A denoising method based on a generalized multidimensional Wiener filter by using a 3rd-order tensor was proposed by Letexier and Bourennane [32]. Furthermore, some denoising method were developed based on basis of tensor decomposition [33]-[35]. Tensor based methods can be represented by tensor decomposition and PCA joint algorithm [36] 


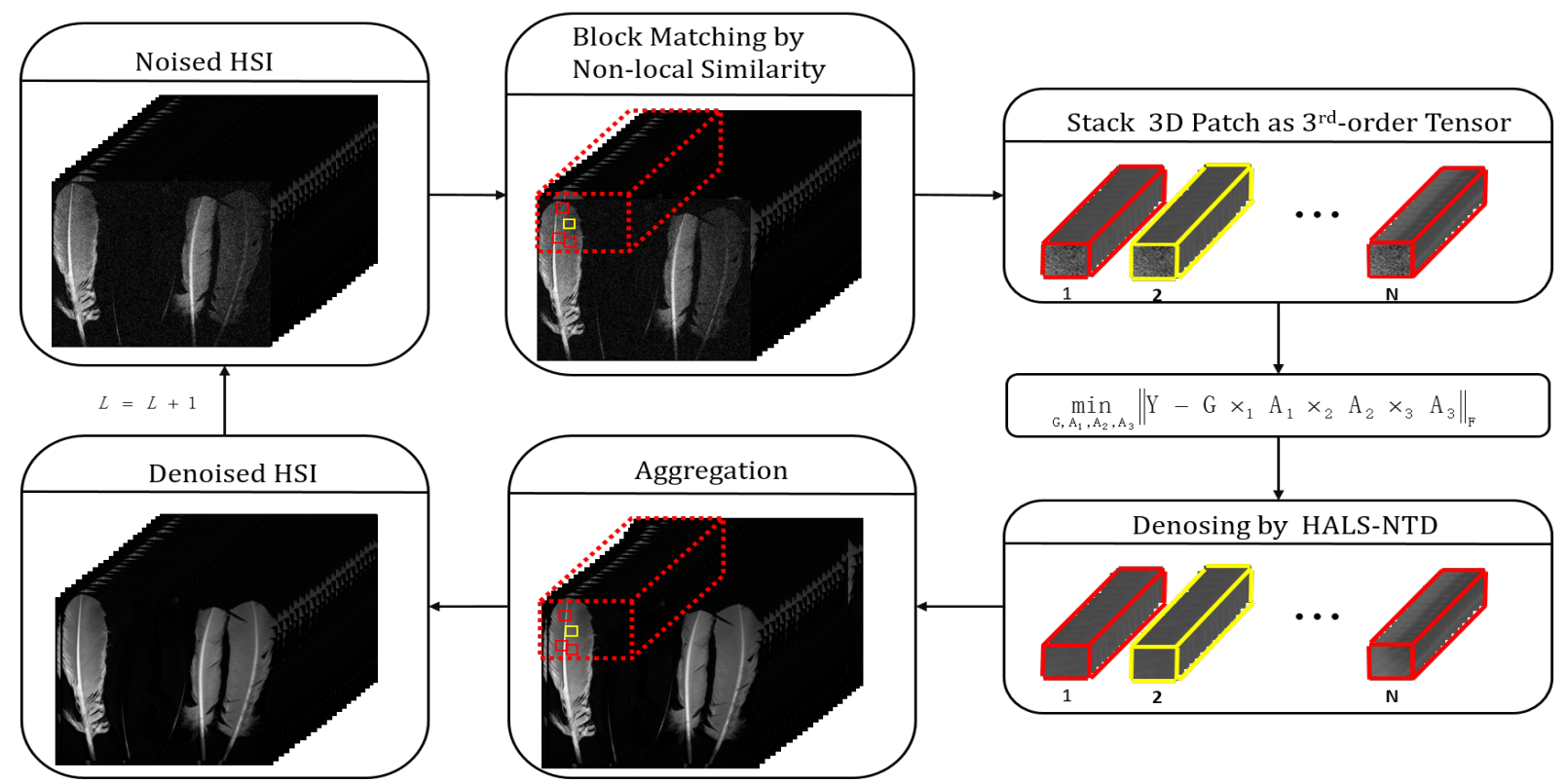

Fig. 1. Overview of the proposed hyperspectral image denoising method. Firstly, image patches extracted from noisy hyperspectral image are stacked as a 3rd-order tensor by non-local similarity matching. Then hierarchical alternative least square based nonnegative tucker decomposition method is applied to get the denoised patches. Finally, the denoised patches are aggregated iteratively to get the clean image.

which take the hyperspectral properties into account. Although utilizing the correlation between different bands of the hyperspectral image, the spatial non-local similarity structures have been ignored, and so these approaches still have not reached the full potential for the denoising problem.

Aiming at the aforementioned issues, we propose a novel denoising method by considering both non-local similarity across the spatial domain and global correlation among the spectral domain. On one hand, a natural scene may contains a collection of patches grouped by non-local similarity over the spatial, composing of homologous aggregation of microstructures. The similarity among those patches is so called non-local similarity. Recovering these patches by several similar non-local patches can utilize the information redundancy across spatial space as well as maintain the texture characteristic of the hyperspectral image. On the other hand, hyperspectral image contains a large amount of spectral redundancy since some neighboring bands are highly correlated. These two kinds of prior knowledge are very helpful for various hyperspectral image recovery problems [37]-[40]. By this way, redundancy in both spatial space and spectral space can be made full use of.

Moreover, tensor decomposition based hyperspectral image denoising method is adopted in our model, which not only emphasizes the similarity among non-local full band patches but also utilizes the redundancy of spectral space. Specifically, we treat each full band patch as a 3rd-order tensor with two spatial modes and a spectral mode, and then build a 3rdorder tensor by stacking all these non-local similar full band patches as shown in Fig. 1. Then the denoising problem can be transformed into tensor recovery by utilizing the inherent spatial and spectral correlation of the full band patch groups.
We solve the tensor recovery problem by adopting a hierarchical alternative least square optimization of nonnegative tensor decomposition and then aggregate the denoised 3D patches in the clean hyperspectral image iteratively. We demonstrate the effectiveness of the proposed method through experimental results on several hyperspectral image datasets. Our method outperforms many state-of-the-art approaches under several quantitative assessments.

The rest of the paper is organized as follows. We illustrate the basic theory of tensor in Section II. In Section III, by exploiting the non-local similarity across spatial space and spectral space, we introduce a denoising algorithm based on tensor decomposition for hyperspectral image. In Section IV, we present and analyze the experimental results. Finally, conclusions are drawn in Section V.

\section{Notation AND PReliminaries of TENSOR}

An $N$-way tensor $\mathcal{X} \in \mathbb{R}^{I_{1} \times I_{2} \times \cdots \times I_{N}}$ has $N$ indices and its elements are denoted by $x_{i_{1} i_{2} \cdots i_{N}}$ where $1 \leq i_{n} \leq I_{n}$, $n=1,2, \ldots, N$.

The scalar product of two tensors $\mathcal{X}, \mathcal{Y}$ is defined as $\langle\mathcal{X}, \mathcal{Y}\rangle=\sum_{i_{1}, i_{2}, \cdots, i_{N}} x_{i_{1} i_{2} \cdots i_{N}} y_{i_{1} i_{2} \cdots i_{N}}$. The Frobenius norm of a tensor $\mathcal{X}$ is given by:

$$
\|\mathcal{X}\|_{F}=\sqrt{\sum_{i_{1}=1}^{I_{1}} \sum_{i_{2}=1}^{I_{2}} \cdots \sum_{i_{N}=1}^{I_{N}} x_{i_{1} i_{2} \cdots i_{N}}^{2}}
$$

Tensor mode-n unfolding: Tensor mode- $n$ unfolding, which is also called tensor matricization, is analogous to vectorizing a matrix. Mode- $n$ unfolding of $\mathcal{X} \in \mathbb{R}^{I_{1} \times I_{2} \times \cdots \times I_{N}}$ re-arranges the elements of $\mathcal{X}$ to 
form a matrix $\mathbf{X}_{(\mathbf{n})} \in \mathbb{R}^{I_{n} \times I_{n+1} I_{n+2} \cdots I_{N} I_{1} I_{2} \cdots I_{n-1}}$, where $I_{n+1} I_{n+2} \cdots I_{N} I_{1} I_{2} \cdots I_{n-1}$ is in cyclic order.

Tensor mode-n matrix product: Mode- $n$ product of a tensor $\mathcal{X} \in \mathbb{R}^{I_{1} \times I_{2} \times \cdots \times I_{N}}$ with a matrix $\mathbf{U} \in \mathbb{R}^{J \times I_{n}}$ is denoted by $\mathcal{X} \times{ }_{n} \mathbf{U}$ and its size is $I_{1} \times \cdots \times I_{n-1} \times J \times I_{n+1} \times \cdots \times I_{N}$. The produced tensor has the same size as $\mathcal{X}$ in each mode, except mode- $n$ whose size is $J$. We have

$$
\left(\mathcal{X} \times{ }_{n} \mathbf{U}\right)_{i_{1} \cdots i_{n-1} j i_{n+1} \cdots i_{N}}=\sum x_{i_{1} i_{2} \cdots i_{N}} u_{j i_{n}}
$$

Multiple matrix mode-n products can be performed in any order,

$$
\left(\mathcal{X} \times{ }_{n} \mathbf{A}\right) \times_{m} \mathbf{B}=\left(\mathcal{X} \times{ }_{m} \mathbf{B}\right) \times_{n} \mathbf{A}
$$

for $m \neq n$.

Tensor mode-n vector product: Mode-n vector product of a tensor $\mathcal{X} \in \mathbb{R}^{I_{1} \times I_{2} \times \cdots \times I_{N}}$ with a vector $v \in \mathbb{R}^{I_{n}}$ is of size $I_{1} \times \cdots \times I_{n-1} \times I_{n+1} \times \cdots \times I_{N}$ and is denoted by $\mathcal{X} \overline{\times}_{n} v$. Element-wise, we have

$$
\left(\mathcal{X} \overline{\times}_{n} v\right)_{i_{1} \cdots i_{n-1} i_{n+1} \cdots i_{N}}=\sum_{i_{n}=1}^{I_{n}} x_{i_{1} i_{2} \cdots i_{N}} v_{i_{n}} .
$$

The basic tensor operations described above show that multiplying a 3-order tensor by a vector in one mode results in a two-way tensor, i.e. a matrix. It is possible to multiple a tensor by a vector in more than one mode as well. Multiplying a 3-order tensor by vectors in two modes results in a 1-order tensor, i.e. a vector. Multiplying in all modes results in a scalar.

Multiplication in all possible modes $(n=1,2, \cdots, N)$ of a tensor $\mathcal{X}$ and a set of matrices $\mathbf{A}^{(n)}$ is defined as

$$
\mathcal{X} \times\{\mathbf{A}\}=\mathcal{X} \times{ }_{1} \mathbf{A}^{(1)} \times{ }_{2} \mathbf{A}^{(2)} \cdots \times{ }_{N} \mathbf{A}^{(N)}
$$

Multiplication of a tensor $\mathcal{X}$ with all but one mode is denoted by

$$
\begin{aligned}
\mathcal{X} \times{ }_{-n}\{\mathbf{A}\}= & \mathcal{X} \times{ }_{1} \mathbf{A}^{(1)} \times_{2} \mathbf{A}^{(2)} \cdots \times_{n-1} \mathbf{A}^{(n-1)} \\
& \times_{n+1} \mathbf{A}^{(n+1)} \cdots \times_{N} \mathbf{A}^{(N)}
\end{aligned}
$$

\section{Hyperspectral IMAGE DENOISING MODEL}

In this section, we first introduce how non-local similar patches are grouped into a 3rd-order tensor. Then we describe the nonnegative tucker decomposition method adopted for the 3rd-order tensor based on hierarchical alternative least square approach.

\section{A. Non-local Similarity Patch Matching}

Acito et al. [41] suggested that signal-independent noise in hyperspectral image can be modeled by Gaussian distribution. A noise free hyperspectral image $\mathcal{X}^{*}$ degraded by additive zero mean Gaussian noise can be modeled as

$$
\mathcal{Y}^{*}=\mathcal{X}^{*}+\mathcal{N}^{*}
$$

where $\mathcal{N}^{*}$ is the noise with a known variance $\sigma^{2}$ and zero mean. The ultimate goal of denoising is to estimate $\widehat{\mathcal{X}}^{*}$ of the clean hyperspectral image $\mathcal{X}^{*}$ from noisy $\mathcal{Y}^{*}$.
Traditional image denoising tasks has generalized the use of non-local similarity based denoising framework. Such framework also can be applied to hyperspectral images. Denote $I_{h}, I_{w}$ and $I_{s}$ as the height and the width in the spatial dimension and the number of spectral bands of an input hyperspectral image respectively, the hyperspectral image can be represented as a 3rd-order tensor $\mathcal{Y}^{*} \in \mathbb{R}^{I_{h} \times I_{w} \times I_{s}}$ with two spatial modes and one spectral mode. A group of $2 \mathrm{D}$ full band patches $\left\{P_{i, j}\right\}_{1 \leq i \leq I_{h}-I_{p}+1,1 \leq j \leq I_{w}-I_{p}+1} \subset \mathbb{R}^{I_{p}^{2} \times I_{s}}$ is built to express the hyperspectral image by sweeping across the image spatially with overlaps, where each band of a full band patch is ordered lexicographically as a column vector. We can now reformulate all full band patches as a group of $2 \mathrm{D}$ patches $\Phi_{\mathcal{Y}}=\left\{\mathcal{Y}_{i} \in \mathbb{R}^{I_{p}^{2} \times I_{s}}\right\}_{i=1}^{S}$, where $S=\left(I_{h}-I_{p}+1\right) \times$ $\left(I_{w}-I_{p}+1\right)$ is the number of patches over the whole hyperspectral image.

For a given local full band patch $P_{\text {ref }}$, we can find a collection of similar full band patches $P_{k}$ from a predefined searching window by utilizing the non-local similarity matching, where $k \in[1, K]$ and $K$ is the number of similar patches. The similarity between $P_{r e f}$ and $P_{k}$ can be formulated as:

$$
\operatorname{Sim}\left(P_{\text {ref }}, P_{k}\right)=\sqrt{\left\|P_{\text {ref }}-P_{k}\right\|^{2}}
$$

The smaller the $\operatorname{Sim}\left(P_{\text {ref }}, P_{k}\right)$, the more similar these two patches turn out to be. Denote $\mathcal{Y} \in \mathbb{R}^{I_{1} \times I_{2} \times I_{3}}$ as the 3rdorder tensor stacked by $P_{\text {ref }}$ and its non-local similar full band patches $P_{k}$ in the search window, where we denote $I_{1}=I_{p}^{2}, I_{2}=I_{s}, I_{3}=K$ for convenient notation. It not only utilizes the non-local similarity across spatial space, but also preserves the global correlation across spectral space.

Then the aforementioned denoising model for the corresponding true non-local similarity full band patches $\mathcal{X}$ from its corrupted $\mathcal{Y}$ is transformed into:

$$
\mathcal{Y}=\mathcal{X}+\mathcal{N}
$$

where $\mathcal{N}$ is the noise tensor of the stacked patches.

The corresponding noise free tensor $\mathcal{X}$ can be estimated from its corrupted $\mathcal{Y}$ by solving the following optimization problem:

$$
\begin{array}{ll}
\min _{\mathcal{G}, \mathbf{A}^{(1)}, \mathbf{A}^{(2)}, \mathbf{A}^{(3)}} & \|\mathcal{Y}-\mathcal{X}\|_{F} \\
\text { s.t. } & \mathcal{X}=\mathcal{G} \times{ }_{1} \mathbf{A}^{(1)} \times{ }_{2} \mathbf{A}^{(2)} \times{ }_{3} \mathbf{A}^{(3)} \\
& \quad \mathbf{A}^{(n) T} \mathbf{A}^{(n)}=\mathbf{I}, n=1,2,3
\end{array}
$$

where $\mathbf{A}^{(1)} \in \mathbb{R}^{I_{1} \times J_{1}}, \mathbf{A}^{(2)} \in \mathbb{R}^{I_{2} \times J_{2}}, \mathbf{A}^{(3)} \in \mathbb{R}^{I_{3} \times J_{3}}$ correspond to the basis vectors in the three modes of $\mathcal{Y}$ with $I_{1} \geq J_{1}, I_{2}>J_{2}$, and $I_{3} \geq J_{3}$. Here $\mathcal{G} \in \mathbb{R}^{J_{1} \times J_{2} \times J_{3}}$ is the so-called core tensor and $J_{1}, J_{2}, J_{3}$ are the ranks responding to three modes. The AIC/MDL criterion [42], [43] can be used to determine the rank for our tensor model. In addition, $I_{2}>J_{2}$ leads to dimensionality reduction in the spectral mode of corrupted tensor $\mathcal{Y}$.

Here we utilize the hierarchical alternative least square (ALS) based nonnegative tucker decomposition to calculate the component $\mathcal{G}, \mathbf{A}^{(1)}, \mathbf{A}^{(2)}, \mathbf{A}^{(3)}$ of the noise free tensor $\mathcal{X}$. 


\section{B. Hierarchical ALS Algorithm for Nonnegative Tucker De- composition}

Due to matrix inversion, standard alternative least square (ALS) [44] suffers from high computational cost for large scale problem, and may not converge to a stable solution. Rather than solving the problem by ALS based tucker decomposition technique, we propose to use a hierarchical alternative least square approach for nonnegative tucker decomposition [45] (referred here as NTD-HALS algorithm), which estimates nonnegative components via a sequential iterative procedure. According to the nonnegative Tucker decomposition, the aforementioned denoising model can be converted into:

$$
\begin{aligned}
\mathcal{Y} & =\mathcal{X}+\mathcal{N} \\
& =\mathcal{G} \times{ }_{1} \mathbf{A}^{(1)} \times{ }_{2} \mathbf{A}^{(2)} \cdots \times_{N} \mathbf{A}^{(N)}+\mathcal{N} \\
& =\sum_{j_{1}=1}^{J_{1}} \sum_{j_{2}=1}^{J_{2}} \cdots \sum_{j_{N}=1}^{J_{N}} g_{j_{1} j_{2} \ldots j_{N}} \mathbf{a}_{j_{1}}^{(1)} \circ \mathbf{a}_{j_{2}}^{(2)} \circ \cdots \circ \mathbf{a}_{j_{N}}^{(N)}
\end{aligned}
$$

where $\mathcal{Y} \in \mathbb{R}_{+}^{I_{1} \times I_{2} \cdots \times I_{N}}$, $\circ$ is the vector outer product, and $\mathcal{X}$ denotes the noise free image to be estimated from the noisy $\mathcal{Y}$, and $\mathcal{G} \in \mathbb{R}_{+}^{J_{1} \times J_{2} \cdots \times J_{N}}$ is the core tensor multiplied by a set of component matrices. $\mathbf{A}^{(n)}=\left[\mathbf{a}_{1}^{(n)}, \mathbf{a}_{2}^{(n)}, \cdots, \mathbf{a}_{J_{n}}^{(n)}\right] \in \mathbb{R}_{+}^{I_{n} \times J_{n}}(n=1,2, \ldots, N)$ represents nonnegative common factors. Instead of estimating the whole factor $\mathbf{A}^{(n)}$, we update each component $\mathbf{a}_{j_{n}}^{(n)}$ of the factor $\mathbf{A}^{(n)}$.

The nonnegative Tucker decomposition is divided into two steps: first we update each nonnegative component $\mathbf{a}_{j_{n}}^{(n)}$ of the $\mathbf{A}^{(n)}$ sequentially, for $n=1,2, \ldots, N$ and $j_{n}=1,2, \ldots, J_{n}$, then we update the core tensor $\mathcal{G}$.

Update factors $\mathbf{A}^{(n)}$ : The denoising model could be split into two parts to estimate $\mathbf{a}_{j_{n}}^{(n)}, n=1,2, \ldots, N$, $j_{n}=1,2, \ldots, J_{n}$. When the specific component $\mathbf{a}_{j_{n}}^{(n)}$ is not involved, the tensor consists of all rank-one tensors can be defined as:

$$
\mathcal{Y}_{j_{n}}^{-}=\sum_{\substack{\overline{\mathbf{k}}=\left[k_{1}, k_{2}, \cdots, k_{N}\right] \\ k_{n} \neq j_{n}}} g_{\overline{\mathbf{k}}} \mathbf{a}_{k_{1}}^{(1)} \circ \mathbf{a}_{k_{2}}^{(2)} \circ \cdots \circ \mathbf{a}_{k_{N}}^{(N)}
$$

When the specific component $\mathbf{a}_{j_{n}}^{(n)}$ is involved, the tensor consists of all rank-one tensors can be denoted by:

$\mathcal{Y}_{j_{n}}^{+}=\sum_{\substack{\overline{\mathbf{k}}=\left[k_{1}, k_{2}, \ldots, k_{N}\right] \\ k_{n}=j_{n}}} g_{\overline{\mathbf{k}}} \mathbf{a}_{k_{1}}^{(1)} \circ \cdots \circ \mathbf{a}_{k_{n-1}}^{(n-1)} \circ \mathbf{a}_{j_{n}}^{(n)} \circ \mathbf{a}_{k_{n+1}}^{(n+1)} \circ \cdots \circ \mathbf{a}_{k_{N}}^{(N)}$

where $k_{n}=1,2, \ldots, J_{n}$ and $\overline{\mathbf{k}}=\left[k_{1}, k_{2}, \ldots, k_{N}\right]$ is the index vector.

The denoising model defined in Eq. (11) can be rewritten using the above notations as follows:

$$
\mathcal{Y}=\mathcal{Y}_{j_{n}}^{-}+\mathcal{Y}_{j_{n}}^{+}+\mathcal{N}
$$

Then we define a residual tensor $\widetilde{\mathcal{Y}}_{j_{n}}$ as:

$$
\begin{aligned}
\widetilde{\mathcal{Y}}_{j_{n}} & =\mathcal{Y}-\mathcal{Y}_{j_{n}}^{-} \\
& =\mathcal{Y}_{j_{n}}^{+}+\mathcal{N} \\
& =\mathcal{G}_{k_{n}=j_{n}} \times{ }_{-n}\{\mathbf{A}\} \times_{n} \mathbf{a}_{j_{n}}^{(n)}+\mathcal{N}
\end{aligned}
$$

Therefore, the component $\mathbf{a}_{j_{n}}^{(n)}$ can be updated from the residual tensor $\widetilde{\mathcal{Y}}_{j_{n}}$ by

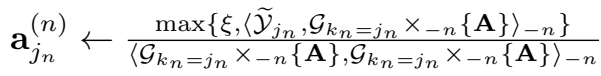

for $j_{n}=1,2, \ldots, J_{n}$ and $n=1,2, \ldots, N$. However, the method is still of high computational complexity due to the computation cost of the residual tensor. We simplify Eq. (16) by replacing the residual tensor $\widetilde{\mathcal{Y}}_{j_{n}}$ via Eq. (15) and algebraic operations. The solution can be transformed into:

$$
\begin{aligned}
\mathbf{a}_{j_{n}}^{(n)} & \leftarrow \frac{\max \left\{\xi,\left\langle\mathcal{G}_{k_{n}=j_{n}} \times{ }_{-n}\{\mathbf{A}\} \times_{n} \mathbf{a}_{j_{n}}^{(n)}+\mathcal{N}, \mathcal{G}_{k_{n}=j_{n}} \times{ }_{-n}\{\mathbf{A}\}\right\rangle_{-n}\right\}}{w_{j_{n}}} \\
& =\max \left\{\xi, \mathbf{a}_{j_{n}}^{(n)}+\frac{1}{w_{j_{n}}}\left\langle\mathcal{N}, \mathcal{G}_{k_{n}=j_{n}} \times_{-n}\{\mathbf{A}\}\right\rangle_{-n}\right\}
\end{aligned}
$$

where $w_{j_{n}}=\left\langle\mathcal{G}_{k_{n}=j_{n}} \times{ }_{-n}\{\mathbf{A}\}, \mathcal{G}_{k_{n}=j_{n}} \times{ }_{-n}\{\mathbf{A}\}\right\rangle_{-n}$ is a scalar computed by current values. On the basis of above transformation, the vector $\mathbf{a}_{j_{n}}^{(n)}$ can be updated by the noise tensor $\mathcal{N}$ and known parameters.

$$
\begin{aligned}
\mathcal{N} & \leftarrow \mathcal{N}+\mathcal{Y}_{j_{n}}^{+}-\widehat{\mathcal{Y}}_{j_{n}}^{+} \\
& =\mathcal{N}+\mathcal{G}_{k_{n}=j_{n}} \times_{-n}\{\mathbf{A}\} \times_{n}\left(\mathbf{a}_{j_{n}}^{(n)}-\widehat{\mathbf{a}}_{j_{n}}^{(n)}\right)
\end{aligned}
$$

where $\widehat{\mathbf{a}}_{j_{n}}^{(n)}$ is updated by the vector $\mathbf{a}_{j_{n}}^{(n)}$, and $\widehat{\mathcal{Y}}_{j_{n}}^{+}$is the updated residual tensor. Based on the above steps, the component $\mathbf{a}_{j_{n}}^{(n)}$ of the $\mathbf{A}^{(n)}$ can be updated sequentially by combining Eq. (17) and Eq. (18).

Update the core tensor $\mathcal{G}$ : Similarly, we attempt to update the core tensor $\mathcal{G}$ item by item so as to update the entry $g_{\overline{\mathbf{j}}}$ of the core tensor $\overline{\mathbf{j}}=\left[j_{1}, j_{2}, \ldots, j_{N}\right]$. We define the tensor $\mathcal{Y}_{\overline{\mathbf{j}}}^{-}$as the tensor excluding the rank one tensors $\mathbf{a}_{j_{1}}^{(1)}, \mathbf{a}_{j_{2}}^{(2)}, \ldots, \mathbf{a}_{j_{N}}^{(N)}$,

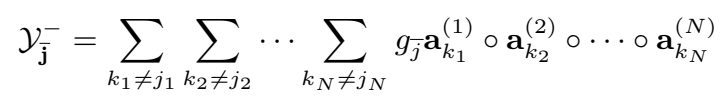

The tensor composed by the components $\mathbf{a}_{j_{1}}^{(1)}, \mathbf{a}_{j_{2}}^{(2)}, \ldots, \mathbf{a}_{j_{N}}^{(N)}$ can be denoted as

$$
\mathcal{Y}_{\overline{\mathbf{j}}}^{+}=g_{\bar{j}} \mathbf{a}_{j_{1}}^{(1)} \circ \mathbf{a}_{j_{2}}^{(2)} \circ \cdots \circ \mathbf{a}_{j_{N}}^{(N)}
$$

According the above notations, the denoising model can be rewritten as

$$
\mathcal{Y}=\mathcal{Y}_{\overline{\mathbf{j}}}^{-}+\mathcal{Y}_{\overline{\mathbf{j}}}^{+}+\mathcal{N}
$$

Then a new residual tensor $\widetilde{\mathcal{Y}}_{\overline{\mathbf{j}}}$ is defined as follows:

$$
\begin{aligned}
\widetilde{\mathcal{Y}}_{\overline{\mathbf{j}}} & =\mathcal{Y}-\mathcal{Y}_{\overline{\mathbf{j}}}^{-} \\
& =\mathcal{Y}_{\overline{\mathbf{j}}}^{+}+\mathcal{N} \\
& =g_{\bar{j}} \mathbf{a}_{j_{1}}^{(1)} \circ \mathbf{a}_{j_{2}}^{(2)} \circ \cdots \circ \mathbf{a}_{j_{N}}^{(N)}+\mathcal{N}
\end{aligned}
$$

Suppose that the vectors $\mathbf{a}_{j_{n}}^{(n)}$ are the unit length vectors, i.e. $\mathbf{a}_{j_{n}}^{(n) T} \mathbf{a}_{j_{n}}^{(n)}=1$. The entry $g_{j}$ can be derived by the following formula:

$$
\begin{aligned}
g_{\bar{j}} & \leftarrow \widetilde{\mathcal{Y}}_{\overline{\mathbf{j}}} \overline{\times}_{1} \mathbf{a}_{j_{1}}^{(1)} \overline{\times}_{2} \mathbf{a}_{j_{2}}^{(2)} \ldots \overline{\times}_{N} \mathbf{a}_{j_{N}}^{(N)} \\
& =\left(\mathcal{Y}_{\overline{\mathbf{j}}}^{+}+\mathcal{N}\right) \overline{\times}_{1} \mathbf{a}_{j_{1}}^{(1)} \overline{\times}_{2} \mathbf{a}_{j_{2}}^{(2)} \cdots \overline{\times}_{N} \mathbf{a}_{j_{N}}^{(N)} \\
& =g_{\bar{j}}+\mathcal{N} \overline{\times}_{1} \mathbf{a}_{j_{1}}^{(1)} \overline{\times}_{2} \mathbf{a}_{j_{2}}^{(2)} \cdots \overline{\times}_{N} \mathbf{a}_{j_{N}}^{(N)}
\end{aligned}
$$


Definite $\widehat{g}_{\bar{j}}$ as the new estimation of $g_{\bar{j}}, \widehat{\mathcal{Y}}_{\overline{\mathbf{j}}}^{+}$to update $\mathcal{Y}_{\overline{\mathbf{j}}}^{+}$. The update strategy of the noise tensor $\mathcal{N}$ can be deduced from Eq. (22).

$$
\begin{aligned}
\mathcal{N} & \leftarrow \mathcal{N}+\mathcal{Y}_{\overline{\mathbf{j}}}^{+}-\widehat{\mathcal{Y}}_{\overline{\mathbf{j}}}^{+} \\
& =\mathcal{N}+\left(g_{\bar{j}}-\widehat{g}_{\bar{j}}\right) \mathbf{a}_{j_{1}}^{(1)} \circ \mathbf{a}_{j_{2}}^{(2)} \circ \cdots \circ \mathbf{a}_{j_{N}}^{(N)}
\end{aligned}
$$

The core tensor $\mathcal{G}$ can be derived by Eq. (23) and Eq. (24) sequentially.

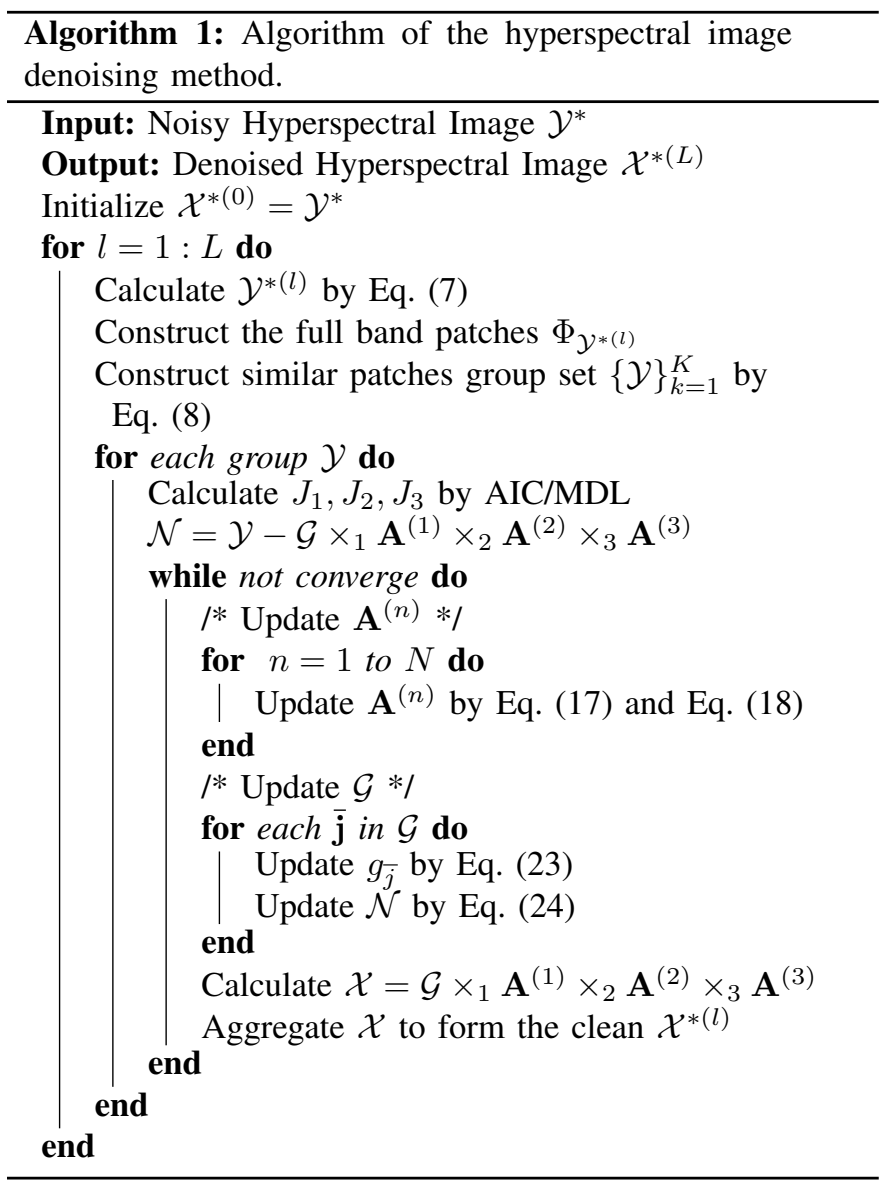

\section{Iterative Denoising Optimization}

Denoising once may not guarantee to get a desired result in practice. Therefore, we propose an iterative denoising strategy for the proposed method, which is a process to take the tradeoff between bias and variance. A single iteration can be done by:

$$
\mathcal{Y}^{*(l)}=\mathcal{X}^{*(l-1)}+\delta\left(\mathcal{Y}^{*}-\mathcal{X}^{*(l-1)}\right)
$$

where $l$ is the iteration number, $\delta \in(0,1)$ is the residual proportionality coefficient. By utilizing Eq. (25) iteratively, the denoised effect could be optimized. Nevertheless, with the increase of iterations, the time consumption tends to become excessively expensive. Hence, the maximum iteration number $L$ should be properly set to balance the time cost and the experiment results.

The algorithm for the proposed hyperspectral image denoising method is summarized in Algorithm 1.

\section{Analysis of Computational Complexity}

Here, we analyze the computational complexity of the proposed method. The computation of non-local similarity patch matching involves full band patch construction and similar patch group set construction. In fact, full band patches can be generated by a sliding window which is related to the patch size and the size of the hyperspectral image in spatial space. Thus this part only considers the time complexity of similar patch group set construction. When the full band patches $\Phi_{\mathcal{Y}^{*}}$ are given, cost of similar patch construction is $\mathcal{O}\left(K S I_{p}^{2} I_{s}\right)$, where $K$ is the number of similar patches, and $S$ is the number of full band patches. Since hierarchical ALS algorithm for nonnegative tucker decomposition is only related to the size of full band patches, the computational complexity of this part is $\mathcal{O}\left(K I_{p}^{2} I_{s}\right)$. Thus, the overall per iteration computational complexity of our method is $\mathcal{O}\left((S+1) K I_{p}^{2} I_{s}\right)$.

\section{EXPERIMENTAL RESULTS}

To demonstrate the effectiveness of the proposed method, we compare it with seven recently developed denoising methods on a simulated hyperspectral image dataset and three hyperspectral image datasets: Columbia dataset [46], Indian Pines dataset [47], Pavia University dataset [48] and Washington DC Mall dataset [47].

The alternative denoising methods include band-wise $\mathrm{K}$ SVD [17] and band-wise BM3D [18]; 3D-cube-based approaches K-SVD [20] and non-local transform domain filter for volumetric data (BM4D) [21] which are extended from 2D methods, state-of-the-art tensor-based approaches low rank tensor approximation (LRTA) [30] and PARAFAC [29], and denoising algorithm via sparse representation and low rank constraint (SPA+LR) [22]. All the parameters of the competing algorithm are optimally assigned or automatically chosen according to the reference papers.

To evaluate the performance of the denoising methods, picture quality indices (PQI), e.g. peak signal-to-noise ratio (PSNR), structure similarity (SSIM) and feature similarity (FSIM), are used to measure the similarity between the denoised image and the original hyperspectral image which is assumed to be the ground truth. PSNR and SSIM evaluate the similarity between the target image and the reference image based on mean square error (MSE) and structural consistency, respectively. SSIM emphasizes the perceptual consistency with the reference image. The higher three measures are, the closer the denoised hyperspectral image is to the ground truth. The denoising performance evaluation metrics for each hyperspectral image are calculated as the average of the band-wise results.

We corrupted a clean hyperspectral image with an additive zero mean Gaussian noise whose $\sigma$ ranges from 10 to 60 . The patch size is set to 6 pixels and the number of similar patches is set to 50 empirically. The size of full band patch searching window depends on the size of input hyperspectral image, ranging from $30 \times 30$ to $80 \times 80$ pixels for all datasets. To demonstrate the influence of window size, we show the average PSNR on the Columbia dataset in Table. II. It shows that the performance will be improved when searching window 

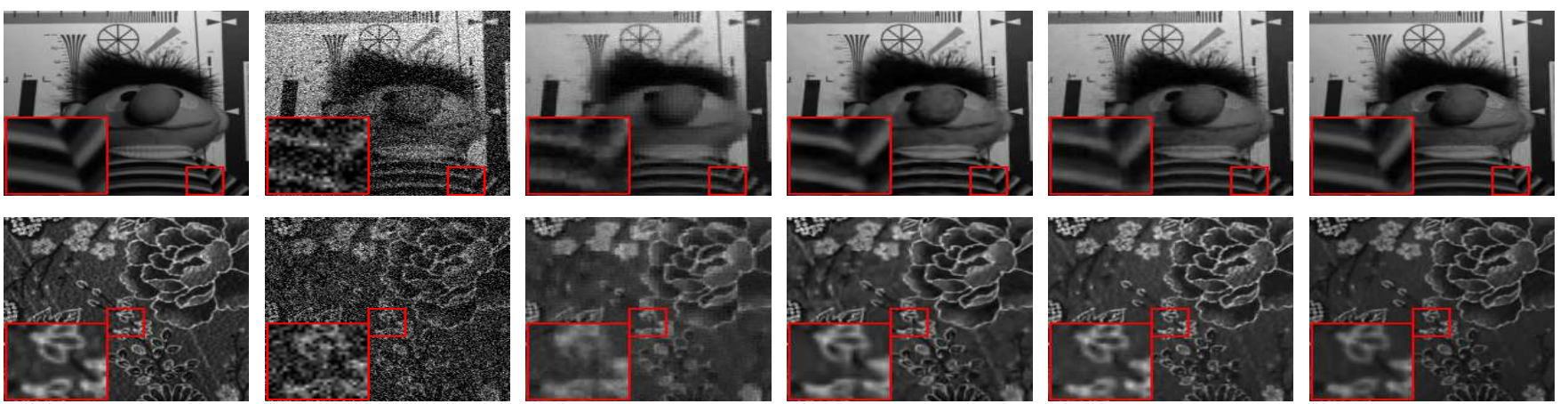

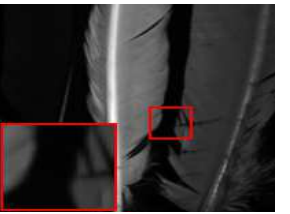

(a) Original

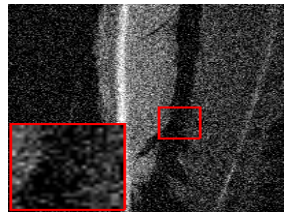

(b) Noisy

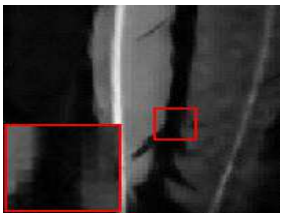

(c) K-SVD

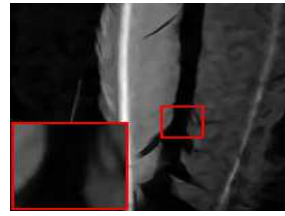

(d) BM4D

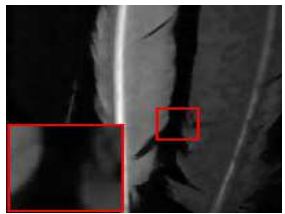

(e) SPA+LR

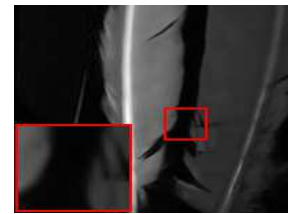

(f) Ours

Fig. 2. (a) The images at band $620 \mathrm{~nm}$ of stuffed toy, cloth and feather; (b) The corresponding images corrupted by the mixture of $\sigma=40$ Gaussian noise; (c)-(f) The restored images obtained by 4 of the comparing 8 denoising method. The demarcated areas in each image is amplified at a 4 times larger scale for easy observation of details.

TABLE I

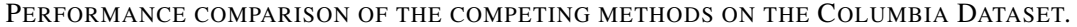

\begin{tabular}{|c|c|c|c|c|c|c|c|c|c|}
\hline \multirow{2}{*}{ Methods } & \multicolumn{3}{|c|}{$\sigma=20$} & \multicolumn{3}{c|}{$\sigma=40$} & \multicolumn{3}{c|}{$\sigma=60$} \\
\cline { 2 - 10 } & PSNR & SSIM & FSIM & PSNR & SSIM & FSIM & PSNR & SSIM & FSIM \\
\hline Noisy & 17.59 & 0.2433 & 0.6253 & 14.27 & 0.1870 & 0.5254 & 11.38 & 0.0962 & 0.4174 \\
PARAFAC & 35.36 & 0.8932 & 0.9599 & 31.52 & 0.7609 & 0.9246 & 29.30 & 0.7857 & 0.8775 \\
LRTA & 37.67 & 0.9437 & 0.9722 & 34.28 & 0.8212 & 0.9223 & 31.53 & 0.8036 & 0.9028 \\
BwK-SVD & 33.95 & 0.8002 & 0.9603 & 29.65 & 0.7157 & 0.9200 & 27.89 & 0.7079 & 0.8564 \\
K-SVD & 36.02 & 0.9397 & 0.9721 & 33.41 & 0.7285 & 0.9203 & 31.90 & 0.8110 & 0.9123 \\
BwBM3D & 38.21 & 0.9497 & 0.9747 & 34.94 & 0.8802 & 0.9358 & 33.84 & 0.8761 & 0.9251 \\
BM4D & 40.57 & 0.9665 & 0.9878 & 37.79 & 0.9105 & 0.9583 & 34.62 & 0.8984 & 0.9399 \\
SPA+LR & 40.96 & 0.9707 & 0.9813 & 38.16 & 0.9334 & 0.9716 & 35.41 & 0.9012 & 0.9421 \\
Ours & $\mathbf{4 2 . 8 7}$ & $\mathbf{0 . 9 8 9 4}$ & $\mathbf{0 . 9 9 4 6}$ & $\mathbf{3 9 . 8 5}$ & $\mathbf{0 . 9 4 7 2}$ & $\mathbf{0 . 9 7 6 0}$ & $\mathbf{3 6 . 2 6}$ & $\mathbf{0 . 9 0 4 8}$ & $\mathbf{0 . 9 5 6 4}$ \\
\hline
\end{tabular}

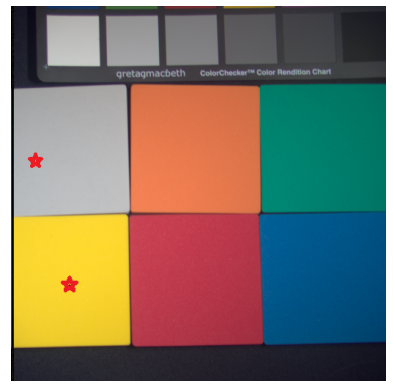

(a)

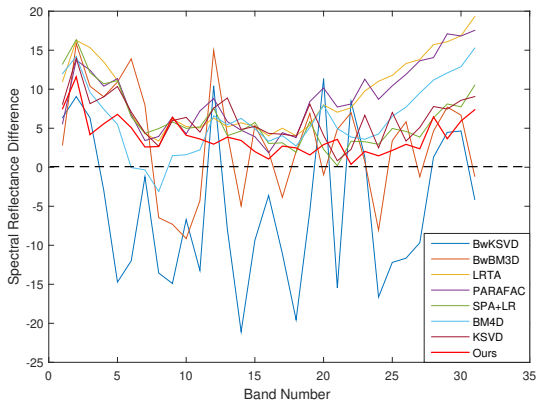

(b)

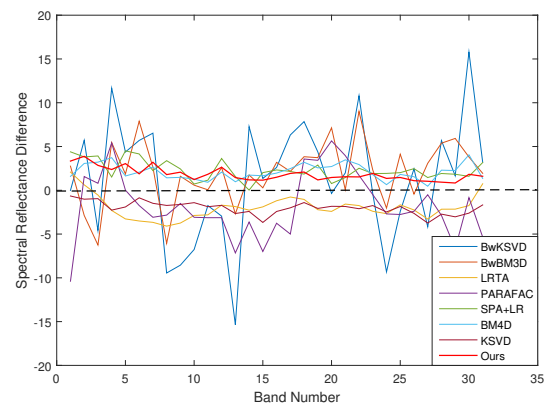

(c)

Fig. 3. (a) Simulated RGB image of sponges; (b) The spectral reflectance difference curves of 8 competing methods at pixel (20, 200); (c)The spectral reflectance difference curves of 8 competing methods at pixel $(80,380)$. The noise free images id corrupted by Gaussian noise of $\sigma=30$. 
TABLE II

PSNR PERFORMANCE FOR DIFFERENT SEARCHING WINDOW SIZES ON THE COLUMBia DATASET.

\begin{tabular}{|c|c|c|c|}
\hline Window Size & $\sigma=20$ & $\sigma=40$ & $\sigma=60$ \\
\hline $30 \times 30$ & 39.63 & 37.14 & 33.93 \\
$40 \times 40$ & 39.45 & 37.59 & 34.12 \\
$50 \times 50$ & 39.72 & 38.07 & 34.51 \\
$60 \times 60$ & 41.54 & 38.46 & 35.39 \\
$70 \times 70$ & 41.65 & 38.68 & 35.41 \\
$80 \times 80$ & 41.59 & 38.66 & 35.27 \\
\hline
\end{tabular}

size increases in a range, and might get worse when searching window becomes too large. The residual proportionality coefficient $\delta$ is set to 0.1 and the maximum iteration number $L$ is determined automatically according to $\sigma$, usually less than three times of $\sigma$ for balancing the time cost and denoising effect.

\section{A. Results on Columbia Dataset}

The Columbia Dataset [46] contains 32 real-world scenes formed by a wide variety of real-world materials and objects. Each hyperspectral image has 31 bands, containing reflectance data collected from $400 \mathrm{~nm}$ to $700 \mathrm{~nm}$ with $10 \mathrm{~nm}$ step. Each band is of $512 \times 512$ in size.

In Fig. 2, we show band at 620nm of stuffed toy, cloth and feather images to demonstrate the performance of various methods. Out method is compared with K-SVD, BM4D, and SPA+LR. Demarcated areas in the scene have been enlarged for 4 times in the figure for easy observation of details. It is obvious that the proposed method outperforms the alternative methods in the recovery of both finer-grained textures and coarser-grained structures.

Table. I lists the performance of all methods, which shows clear advantage of the proposed method over alternative. To further illustrate such superiority, we demonstrate in Fig. 3 the spectral reflectance difference curves of all competing methods using image sponges. Spectral reflectance difference curves of a hyperspectral image denoising method at two spatial locations are obtained by sequentially interpolating 31 elements of the deviation between the restored and the clean hyperspectral image along their spectral domain. The curves of our proposed method are smoother than other methods and the mean square error is smaller. This further proves that our method achieves better approximation than alternative methods.

\section{B. Results on Indian Pines Dataset}

Indian Pines scene was collected by the AVIRIS sensor over the Indian Pines test site in North-western Indiana. The image consists of $145 \times 145$ pixels and 224 spectral bands.

In Fig. 5, we show the denoising performance at the $45^{\text {th }}$ band of the Indian Pines dataset under Gaussian noise with $\sigma$ ranging from 10 to 50 . Our method is capable of properly removing the different degrees of Gaussian noises while finely preserving the underlying structure in the hyperspectral image. More noise is added, the worse is the denoising effect. From
Table III, we can infer that the proposed method evidently outperforms all other competing methods with $\sigma=20,30,40,50$ respectively.

For ease of observation, we illustrate an example image located at the $45^{t h}$ band of Indian Pines dataset in Fig. 4. Among alternative methods, BM4D and SPA+LR perform comparatively better in structure preserving. However, the images restored by them have more blurred edges than those obtained by our method. Compared with traditional non-local similarity based and dictionary learning based methods, our method restores the corrupted image preferably because it utilizes properties in both spectral and spatial domains. Fig. 6 shows the denoising performance of Indian Pines dataset for band 1 to 60 , in the case of $\sigma=20$.

\section{Results on Pavia University Dataset}

Hyperspectral image acquired using ROSIS over the Pavia University is chosen for this experiment. Pavia University dataset contains 103 spectral bands with a spectral coverage ranging from 0.43 to $0.86 \mu \mathrm{m}$ and a spatial resolution of 1.3 meter per pixel. This image is of the size $610 \times 340 \times 103$.

Table IV lists the performance of all comparing methods on the Pavia University dataset corresponding to various degrees of noises. For each noise parameter, the PQI value for each competing HSI denoising method has been calculated and recorded. From these results, the advantage of the proposed method can be clearly observed. We show in Fig. 7 the $15^{\text {th }}$ band with $340 \times 340$ window size extracted from the Pavia University dataset for further assessment of our method. It is obvious that the proposed method outperforms the comparing methods. The PSNR analysis of each spectrum presented in Fig. 8 illustrates that the denoising effect of the proposed method at each band of the Pavia University Dataset with the noise of $\sigma=30$ is superior than any other comparing methods. This proves again that combining non-local similarity and tensor decomposition can preserve both spatial information and spectral information, which leads to prominent advantages in denoising effect.

\section{Results on Washington DC Mall Dataset}

Washington DC Mall Dataset was collected using an airborne system containing 210 spectral bands from 0.4 to 2.4 $\mu m$ in the visible to infrared spectrum. It contains 1028 scan lines with 307 pixels in each scan line.

We used a patch of size $300 \times 300 \times 190$ in the Washington DC image for experiments. The PSNR, SSIM and FSIM are presented in Table V. This table shows that, of the nine methods, the proposed non-local similarity based denoising algorithm still works very effectively. The results of all methods at the $15^{\text {th }}$ band of the Washington DC Mall image are shown in Fig. 9. It can be concluded that the image restored by our method is capable of properly removing the Gaussian noise while finely preserving the structure underlying in the hyperspectral image. Not only the noise is well suppressed, but also the detailed information, both in edges and textures, is well preserved. Comparison of PSNR values among various methods on the Washington DC Dataset in Fig. 10 also shows 


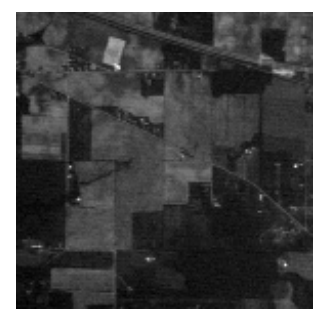

(a) Original

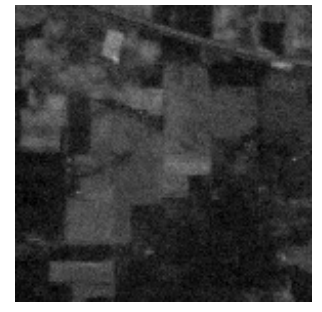

(f) K-SVD [20]

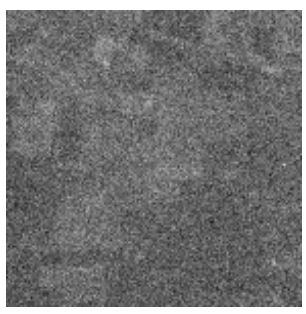

(b) Noisy

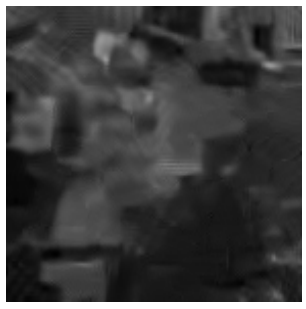

(g) BwBM3D [18]

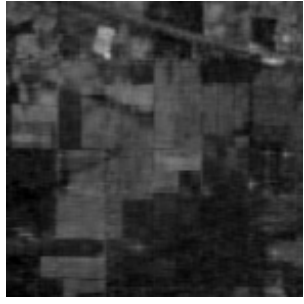

(c) LRTA [30]

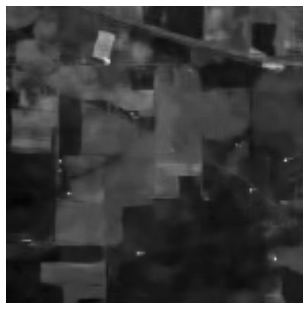

(h) BM4D [21]

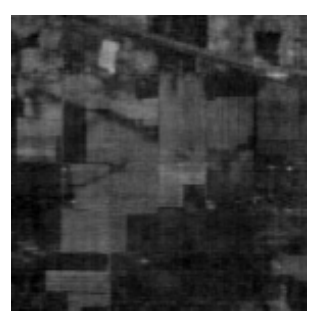

(d) PARAFAC [29]

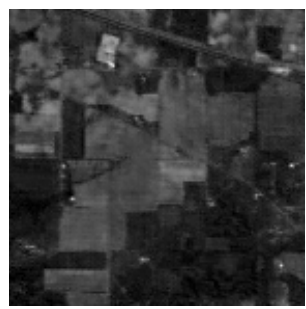

(i) SPA+LR [22]

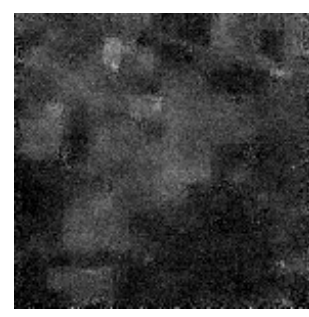

(e) BwK-SVD [17]

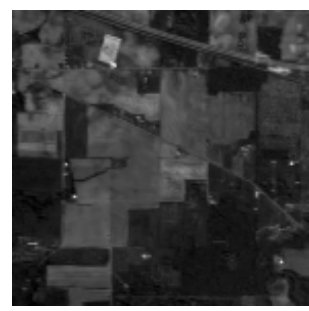

(j) Ours

Fig. 4. (a) The Original image located at the $45^{t h}$ band in the Indian Pines dataset; (b) The corrupted image by Gaussian noise of $\sigma=20$; (c)-(f) The denoised hyperspectral images generated by the alternative methods.

TABLE III

PERFORMANCE COMPARISON OF THE COMPETING METHODS ON THE INDIAN PINES DATASET

\begin{tabular}{|c|c|c|c|c|c|c|c|c|c|c|c|c|}
\hline \multirow{2}{*}{ Methods } & \multicolumn{3}{|c|}{$\sigma=20$} & \multicolumn{3}{c|}{$\sigma=30$} & \multicolumn{3}{c|}{$\sigma=40$} & \multicolumn{3}{c|}{$\sigma=50$} \\
\cline { 2 - 12 } & PSNR & SSIM & FSIM & PSNR & SSIM & FSIM & PSNR & SSIM & FSIM & PSNR & SSIM & FSIM \\
\hline Noisy & 22.134 & 0.394 & 0.730 & 18.617 & 0.117 & 0.407 & 16.113 & 0.081 & 0.352 & 14.175 & 0.054 & 0.302 \\
LRTA [30] & 38.640 & 0.902 & 0.936 & 36.543 & 0.854 & 0.905 & 34.832 & 0.826 & 0.896 & 34.328 & 0.826 & 0.889 \\
PARAFAC [29] & 38.877 & 0.904 & 0.933 & 35.225 & 0.830 & 0.891 & 29.536 & 0.582 & 0.777 & 27.479 & 0.463 & 0.716 \\
BwK-SVD [17] & 30.584 & 0.678 & 0.863 & 28.947 & 0.582 & 0.775 & 28.129 & 0.529 & 0.760 & 27.349 & 0.486 & 0.745 \\
K-SVD [20] & 35.329 & 0.836 & 0.893 & 33.533 & 0.769 & 0.860 & 33.459 & 0.781 & 0.867 & 22.116 & 0.203 & 0.535 \\
BwBM3D [18] & 36.736 & 0.867 & 0.884 & 35.174 & 0.833 & 0.863 & 33.358 & 0.794 & 0.854 & 33.002 & 0.774 & 0.838 \\
BM4D [21] & 38.642 & 0.906 & 0.917 & 36.826 & 0.874 & 0.898 & 35.105 & 0.845 & 0.892 & 34.069 & 0.819 & 0.881 \\
SPA+LR [22] & 41.143 & 0.931 & 0.951 & 38.301 & 0.903 & 0.930 & 36.328 & 0.838 & 0.899 & 35.523 & 0.869 & 0.914 \\
Ours & $\mathbf{4 1 . 9 3 7}$ & $\mathbf{0 . 9 4 6}$ & $\mathbf{0 . 9 7 5}$ & $\mathbf{3 9 . 0 2 6}$ & $\mathbf{0 . 9 1 2}$ & $\mathbf{0 . 9 3 9}$ & $\mathbf{3 7 . 0 1 2}$ & $\mathbf{0 . 8 6 2}$ & $\mathbf{0 . 9 1 7}$ & $\mathbf{3 5 . 9 7 2}$ & $\mathbf{0 . 8 9 3}$ & $\mathbf{0 . 9 2 6}$ \\
\hline
\end{tabular}

TABLE IV

PERFormance COMPARISON OF THE COMPETING METHOdS ON THE PAVIA UniVERSity DATASET.

\begin{tabular}{|c|c|c|c|c|c|c|c|c|c|c|c|c|}
\hline \multirow{2}{*}{ Methods } & \multicolumn{3}{|c|}{$\sigma=20$} & \multicolumn{3}{|c|}{$\sigma=30$} & \multicolumn{3}{c|}{$\sigma=40$} & \multicolumn{3}{c|}{$\sigma=50$} \\
\cline { 2 - 11 } & PSNR & SSIM & FSIM & PSNR & SSIM & FSIM & PSNR & SSIM & FSIM & PSNR & SSIM & FSIM \\
\hline Noisy & 22.143 & 0.386 & 0.710 & 18.616 & 0.190 & 0.538 & 16.122 & 0.164 & 0.519 & 14.179 & 0.087 & 0.399 \\
LRTA [30] & 33.158 & 0.821 & 0.917 & 31.432 & 0.766 & 0.886 & 30.069 & 0.714 & 0.856 & 29.157 & 0.674 & 0.837 \\
PARAFAC [29] & 29.759 & 0.734 & 0.851 & 29.556 & 0.718 & 0.847 & 29.286 & 0.697 & 0.842 & 28.951 & 0.672 & 0.834 \\
BwK-SVD [17] & 28.335 & 0.687 & 0.856 & 26.626 & 0.596 & 0.812 & 25.872 & 0.504 & 0.623 & 24.715 & 0.436 & 0.517 \\
K-SVD [20] & 34.372 & 0.917 & 0.942 & 32.319 & 0.847 & 0.893 & 30.410 & 0.828 & 0.903 & 31.328 & 0.804 & 0.862 \\
BwBM3D [18] & 31.994 & 0.734 & 0.851 & 30.338 & 0.770 & 0.853 & 29.196 & 0.729 & 0.829 & 28.461 & 0.702 & 0.805 \\
BM4D [21] & 36.342 & 0.931 & 0.958 & 34.491 & 0.873 & 0.920 & 32.450 & 0.861 & 0.911 & 31.981 & 0.814 & 0.880 \\
SPA+LR [22] & 37.542 & 0.948 & 0.975 & 34.503 & 0.875 & 0.933 & 33.519 & 0.893 & 0.942 & 32.187 & 0.819 & 0.901 \\
Ours & $\mathbf{3 9 . 9 3 7}$ & $\mathbf{0 . 9 6 9}$ & $\mathbf{0 . 9 8 4}$ & $\mathbf{3 7 . 7 2 8}$ & $\mathbf{0 . 9 5 6}$ & $\mathbf{0 . 9 6 1}$ & $\mathbf{3 6 . 0 1 2}$ & $\mathbf{0 . 9 2 7}$ & $\mathbf{0 . 9 5 6}$ & $\mathbf{3 4 . 4 7 2}$ & $\mathbf{0 . 9 0 3}$ & $\mathbf{0 . 9 2 8}$ \\
\hline
\end{tabular}

TABLE V

PERFORMANCE COMPARISON OF THE COMPETING METHODS ON THE WASHINGTON DC MALL DATASET.

\begin{tabular}{|c|c|c|c|c|c|c|c|c|c|c|c|c|}
\hline \multirow{2}{*}{ Methods } & \multicolumn{3}{|c|}{$\sigma=20$} & \multicolumn{3}{c|}{$\sigma=30$} & \multicolumn{3}{c|}{$\sigma=40$} & \multicolumn{3}{c|}{$\sigma=50$} \\
\cline { 2 - 11 } & PSNR & SSIM & FSIM & PSNR & SSIM & FSIM & PSNR & SSIM & FSIM & PSNR & SSIM & FSIM \\
\hline Noisy & 22.139 & 0.392 & 0.688 & 18.618 & 0.249 & 0.528 & 16.119 & 0.168 & 0.506 & 14.184 & 0.120 & 0.449 \\
LRTA [30] & 35.672 & 0.909 & 0.947 & 33.368 & 0.862 & 0.919 & 31.735 & 0.816 & 0.893 & 30.448 & 0.770 & 0.870 \\
PARAFAC [29] & 31.674 & 0.838 & 0.900 & 31.307 & 0.824 & 0.893 & 30.836 & 0.805 & 0.885 & 30.159 & 0.776 & 0.874 \\
BwK-SVD [17] & 28.687 & 0.672 & 0.845 & 27.253 & 0.580 & 0.804 & 26.408 & 0.524 & 0.780 & 25.809 & 0.488 & 0.766 \\
K-SVD [20] & 36.132 & 0.939 & 0.902 & 32.426 & 0.865 & 0.902 & 34.41 & 0.728 & 0.878 & 31.616 & 0.846 & 0.900 \\
BwBM3D [18] & 31.072 & 0.826 & 0.884 & 29.475 & 0.765 & 0.843 & 28.384 & 0716 & 0.812 & 27.668 & 0.678 & 0.779 \\
BM4D [21] & 41.056 & 0.951 & 0.928 & 38.770 & 0.927 & 0.908 & 37.156 & 0.907 & 0.891 & 35.909 & 0.887 & 0.878 \\
SPA+LR [22] & 40.967 & 0.960 & 0.945 & 39.564 & 0.941 & 0.932 & 38.072 & 0.917 & 0.911 & 36.742 & 0.894 & 0.894 \\
Ours & $\mathbf{4 2 . 8 7 0}$ & $\mathbf{0 . 9 7 4}$ & $\mathbf{0 . 9 5 6}$ & $\mathbf{4 0 . 3 7 1}$ & $\mathbf{0 . 9 5 4}$ & $\mathbf{0 . 9 3 9}$ & $\mathbf{3 9 . 5 6}$ & $\mathbf{0 . 9 3 8}$ & $\mathbf{0 . 9 2 6}$ & $\mathbf{3 7 . 2 6 3}$ & $\mathbf{0 . 9 1 7}$ & $\mathbf{0 . 9 2 2}$ \\
\hline
\end{tabular}




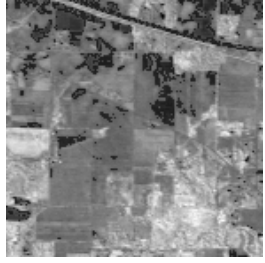

(a) Original

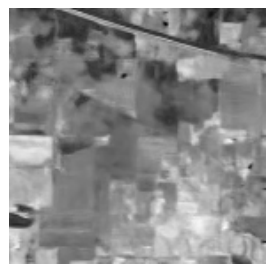

(d) $\sigma=30$

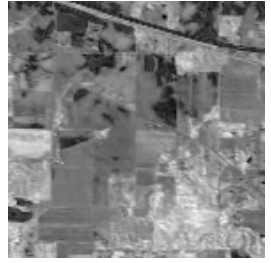

(b) $\sigma=10$

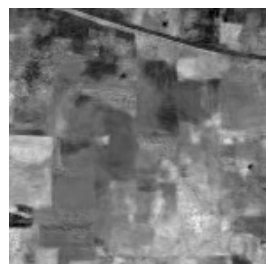

(e) $\sigma=40$

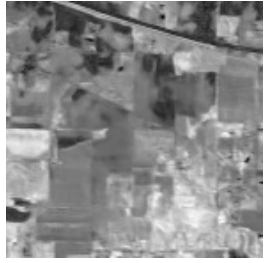

(c) $\sigma=20$

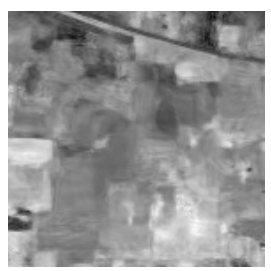

(f) $\sigma=50$
Fig. 5. (a) The original image located at the $20^{t h}$ band in the Indian Pines dataset; (b)-(f) The denoised images obtained by our method with each images corrupted by different Gaussian noises ranging from 10 to 50 .

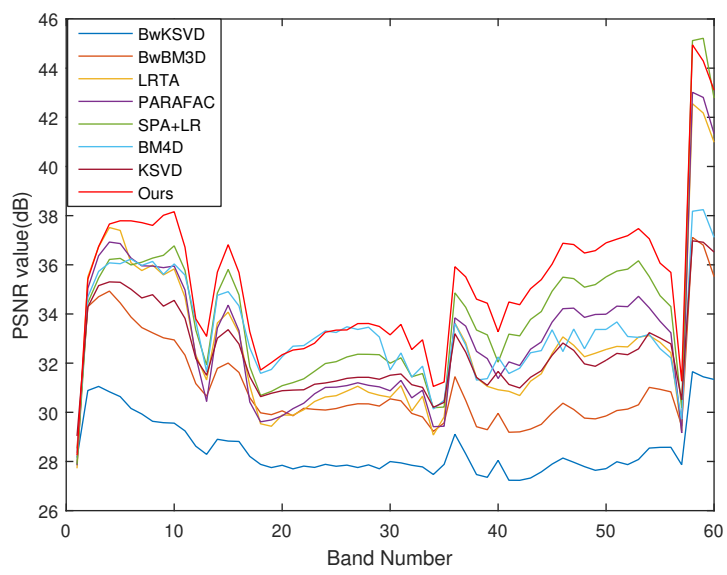

Fig. 6. Comparison of PSNR values among various methods on the Indian Pines Dataset with $\sigma=20$.

that the proposed algorithm achieves higher performance than others in the case of $\sigma=30$. These results demonstrate the effectiveness of our denoising method.

\section{CONCLuSion}

In this paper, we have introduced a novel hyperspectral image denoising method based on tensor decomposition which utilizes both non-local similarity across the spatial space and global redundancy along the spectral space. Unlike previous vector or matrix based approaches, the proposed method considers the hyperspectral image as a 3D tensor and simultaneously exploits the spatial-spectral information of the hyperspectral image, thus boosts the denoising performance. We solved this model by a hierarchical ALS based nonnegative tucker decomposition rather than traditional approach so as to achieve better denoising effect. Experiments on three real hyperspectral image datasets demonstrated that the proposed method outperforms several well-known denoising approaches in terms of both visual quality and PSNR metrics. Last but not least, the proposed tensor-based framework can also be applied to other hyperspectral image processing problems (e.g., reconstruction and unmixing) and enhance the performance in those applications. In the optimization step, though each component is updated sequentially, the time complexity of our solution still can be improved, which will be our future research task.

\section{ACKNOWLEDGMENT}

This work is supported by NSFC project No.61370123, No.61772057 and BNSF project No.4162037. It is also supported by funding from State Key Lab of Software Development Environment in Beihang University.

\section{REFERENCES}

[1] U. Heiden, K. Segl, S. Roessner, and H. Kaufmann, "Determination of robust spectral features for identification of urban surface materials in hyperspectral remote sensing data," Remote Sensing of Environment, vol. 111 , no. 4, pp. 537-552, 2007.

[2] Xiao Bai, Huigang Zhang, and Jun Zhou, "Vhr object detection based on structural feature extraction and query expansion," IEEE Transactions on Geoscience and Remote Sensing, vol. 52, no. 10, pp. 6508-6520, 2014.

[3] C. Li, X. Chen, and Y. Jiang, "On diverse noises in hyperspectral unmixing," IEEE Transactions on Geoscience and Remote Sensing, vol. 53, no. 10 , pp. 5388-5402, 2015.

[4] J. Bioucas-Dias, A. Plaza, N. Dobigeon, M. Parente, Q. Du, P. Gader, and J. Chanussot, "Hyperspectral unmixing overview: Geometrical, statistical, and sparse regression-based approaches," IEEE Journal of Selected Topics in Applied Earth Observations and Remote Sensing, vol. 5, no. 2, pp. 354-379, 2012.

[5] Tong Lei, Jun Zhou, Yuntao Qian, Bai Xiao, and Yongsheng Gao, "Nonnegative-matrix-factorization-based hyperspectral unmixing with partially known endmembers," IEEE Transactions on Geoscience and Remote Sensing, vol. 54, no. 11, pp. 6531-6544, 2016.

[6] Huigang Zhang, Bai Xiao, Huaxin Zheng, Huijie Zhao, Jun Zhou, Cheng Jian, and Hanqing Lu, "Hierarchical remote sensing image analysis via graph laplacian energy," IEEE Geoscience and Remote Sensing Letters, vol. 10, no. 2, pp. 396-400, 2012.

[7] Y. Tarabalka and A. Rana, "Graph-cut-based model for spectral-spatial classification of hyperspectral images," in IEEE Geoscience and Remote Sensing Symposium, 2014, pp. 3418-3421.

[8] X. Kang, S. Li, and J. Benediktsson, "Spectral-spatial hyperspectral image classification with edge-preserving filtering," IEEE Transactions on Geoscience and Remote Sensing, vol. 52, no. 5, pp. 2666-2677, 2014.

[9] L. Mou, P. Ghamisi, and X. Zhu, "Deep recurrent neural networks for hyperspectral image classification," IEEE Transactions on Geoscience and Remote Sensing, vol. PP, no. 99, pp. 1-17, 2017.

[10] Cheng Yan, Xiao Bai, Peng Ren, Lu Bai, Wenzhong Tang, and Jun Zhou, "Band weighting via maximizing interclass distance for hyperspectral image classification," IEEE Geoscience and Remote Sensing Letters, vol. 13, no. 7, pp. 922-925, 2016.

[11] N. Nasrabadi, "Hyperspectral target detection: An overview of current and future challenges," IEEE Signal Processing Magazine, vol. 31, no. 1, pp. 34-44, 2014.

[12] S. Matteoli, M. Diani, and J. Theiler, "An overview of background modeling for detection of targets and anomalies in hyperspectral remotely sensed imagery," IEEE Journal of Selected Topics in Applied Earth Observations and Remote Sensing, vol. 7, no. 6, pp. 2317-2336, 2014.

[13] J. Yin, C. Gao, and X. Jia, "A new target detector for hyperspectral data using cointegration theory," IEEE Journal of Selected Topics in Applied Earth Observations and Remote Sensing, vol. 6, no. 2, pp. 638-643, 2013.

[14] Xiao Bai, Zhouxiao Guo, Yanyang Wang, Zhihong Zhang, and Jun Zhou, "Semisupervised hyperspectral band selection via spectralcspatial hypergraph model," IEEE Journal of Selected Topics in Applied Earth Observations and Remote Sensing, vol. 8, no. 6, pp. 2774-2783, 2015.

[15] A. Banerjee, P. Burlina, and J. Broadwater, "Hyperspectral video for illumination-invariant tracking," in First Workshop on Hyperspectral Image and Signal Processing: Evolution in Remote Sensing, 2009, pp. $1-4$. 


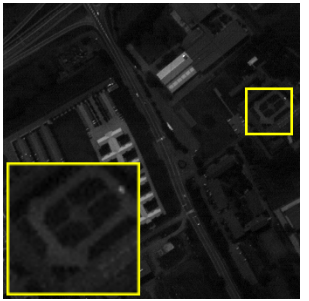

(a) Original

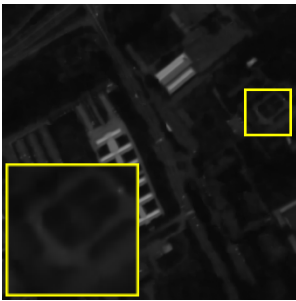

(f) K-SVD [20]

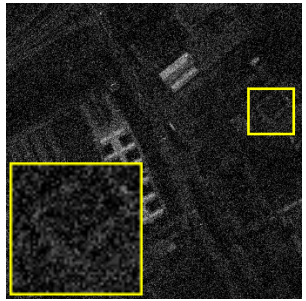

(b) Noisy

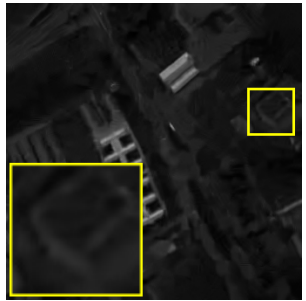

(g) BwBM3D [18]

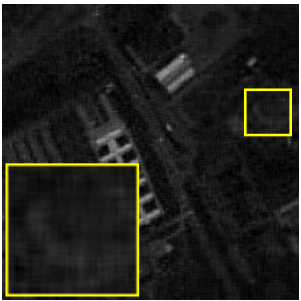

(c) LRTA [30]

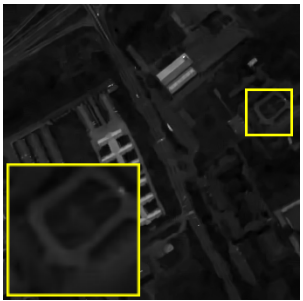

(h) BM4D [21]

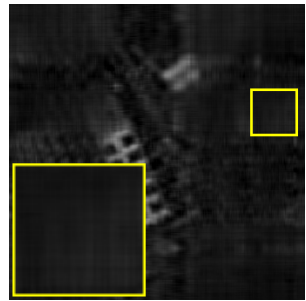

(d) PARAFAC [29]

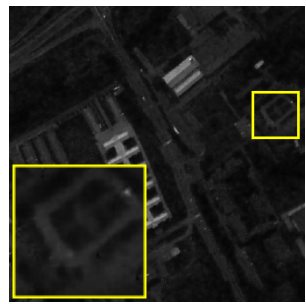

(i) SPA+LR [22]

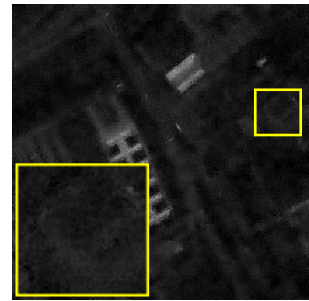

(e) BwK-SVD [17]

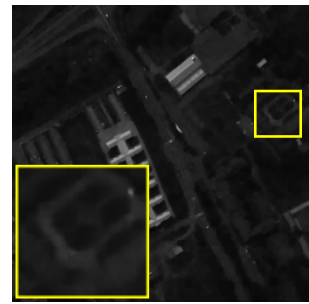

(j) Ours

Fig. 7. (a) The Original image located at the $15^{\text {th }}$ band in the Pavia University dataset; (b) The corrupted image with Gaussian noise of $\sigma=30$; (c)-(f) The denoised hyperspectral images obtained by the comparing denoising methods. Image patches at the bottom-left are zoomed-in views from the top-right small patches.

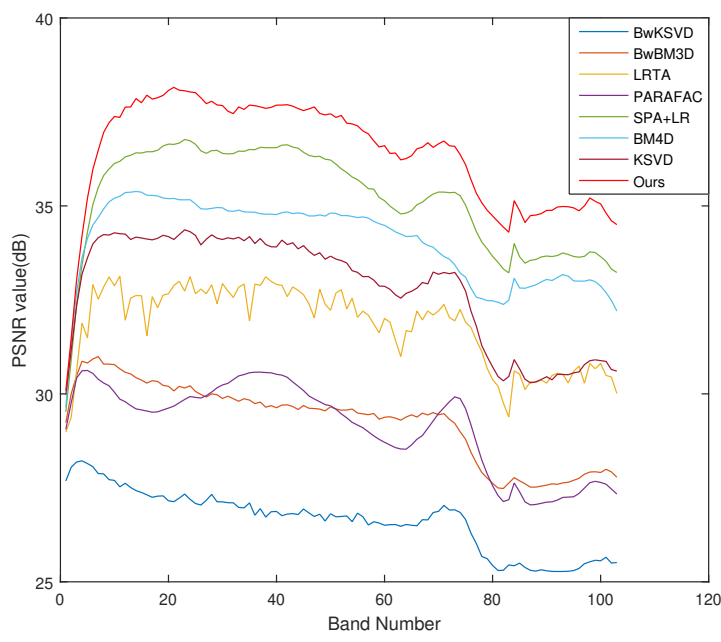

Fig. 8. Comparison of PSNR values among various methods on the Pavia University Dataset with $\sigma=30$.

[16] A. Buades, B. Coll, and J. Morel, "A non-local algorithm for image denoising," in IEEE Computer Society Conference on Computer Vision and Pattern Recognition, 2005, pp. 60-65.

[17] M. Aharon, M. Elad, and A. Bruckstein, "K-SVD: An algorithm for designing overcomplete dictionaries for sparse representation," IEEE Transactions on Signal Processing, vol. 54, no. 11, pp. 4311-4322, 2006.

[18] K. Dabov, A. Foi, V. Katkovnik, and K. Egiazarian, "Image denoising by sparse 3-D transform-domain collaborative filtering.," IEEE Transactions on Image Processing A Publication of the IEEE Signal Processing Society, vol. 16, no. 8, pp. 2080-2095, 2007.

[19] J. Manjon, P. Coupe, L. Marti-Bonmati, D. Collins, and M. Robles, "Adaptive non-local means denoising of MR images with spatially varying noise levels," Journal of Magnetic Resonance Imaging, vol. 31, no. 1, pp. 192C203, 2010.

[20] M. Elad and M. Aharon, "Image denoising via sparse and redundant representations over learned dictionaries," IEEE Transactions on Image Processing A Publication of the IEEE Signal Processing Society, vol. 15 , no. 12 , pp. $3736-3745,2006$.
[21] A. Foi, "Nonlocal transform-domain denoising of volumetric data with groupwise adaptive variance estimation," vol. 8296, no. 3, pp. -, 2012.

[22] Yo. Zhao and J. Yang, "Hyperspectral image denoising via sparse representation and low-rank constraint," IEEE Transactions on Geoscience and Remote Sensing, vol. 53, no. 1, pp. 296-308, 2015.

[23] J. Yang, Y. Zhao, C. Chan, and S. Kong, "Coupled sparse denoising and unmixing with low-rank constraint for hyperspectral image," IEEE Transactions on Geoscience and Remote Sensing, vol. 54, no. 3, pp. 1818-1833, 2016.

[24] T. Lu, S. Li, L. Fang, Y. Ma, and J. Benediktsson, "Spectral-spatial adaptive sparse representation for hyperspectral image denoising," IEEE Transactions on Geoscience and Remote Sensing, vol. 54, no. 1, pp. 373-385, 2016.

[25] S. Zubair and W. Wang, "Tensor dictionary learning with sparse TUCKER decomposition," in International Conference on Digital Signal Processing, 2013, pp. 1-6.

[26] J. Liu, P Musialski, P Wonka, and J. Ye, "Tensor completion for estimating missing values in visual data," IEEE Transactions on Pattern Analysis and Machine Intelligence, vol. 35, no. 1, pp. 208-20, 2013.

[27] P. Yi, D. Meng, Z. Xu, C. Gao, Y. Yi, and B. Zhang, "Decomposable nonlocal tensor dictionary learning for multispectral image denoising," in Computer Vision and Pattern Recognition, 2014, pp. 2949-2956.

[28] A. Rajwade, A. Rangarajan, and A. Banerjee, "Image denoising using the higher order singular value decomposition," IEEE Transactions on Pattern Analysis and Machine Intelligence, vol. 35, no. 4, pp. 849-862, 2013.

[29] X. Liu, S. Bourennane, and C. Fossati, "Denoising of hyperspectral images using the PARAFAC model and statistical performance analysis," IEEE Transactions on Geoscience and Remote Sensing, vol. 50, no. 10 , pp. 3717-3724, 2012.

[30] N. Renard, S. Bourennane, and J. Blanc-Talon, "Denoising and dimensionality reduction using multilinear tools for hyperspectral images," IEEE Geoscience and Remote Sensing Letters, vol. 5, no. 2, pp. 138$142,2008$.

[31] T. Kolda and B. Bader, "Tensor decompositions and applications," Siam Review, vol. 51, no. 3, pp. 455-500, 2009.

[32] D. Letexier and S. Bourennane, "Noise removal from hyperspectral images by multidimensional filtering," IEEE Transactions on Geoscience and Remote Sensing, vol. 46, no. 7, pp. 2061-2069, 2008.

[33] A. Karami, M. Yazdi, and A. Asli, "Noise reduction of hyperspectral images using kernel non-negative tucker decomposition," IEEE Journal of Selected Topics in Signal Processing, vol. 5, no. 3, pp. 487-493, 2011.

[34] X. Guo, X. Huang, L. Zhang, and L. Zhang, "Hyperspectral image noise reduction based on rank-1 tensor decomposition," ISPRS Journal of Photogrammetry and Remote Sensing, vol. 83, no. 9, pp. 50-63, 2013.

[35] L. Fang, N. He, and H. Lin, "CP tensor-based compression of 


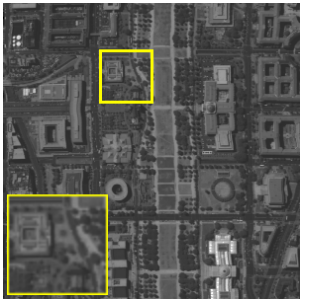

(a) Original

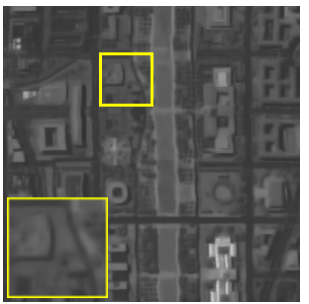

(f) K-SVD [20]

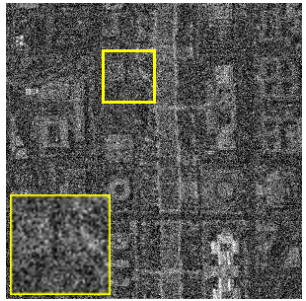

(b) Noisy

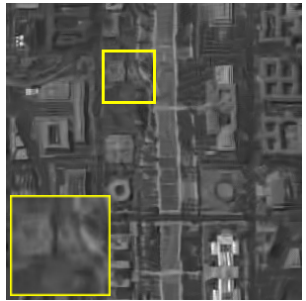

(g) BwBM3D [18]

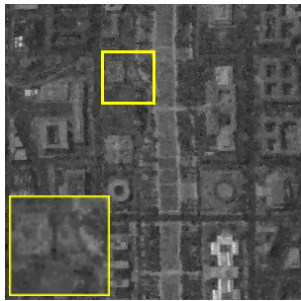

(c) LRTA [30]

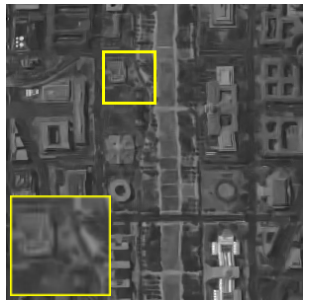

(h) BM4D [21]

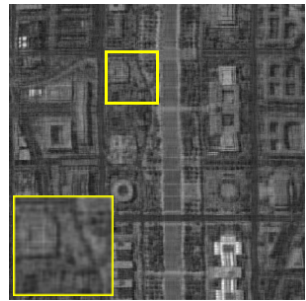

(d) PARAFAC [29]

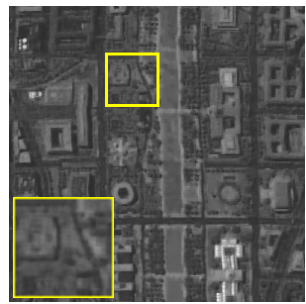

(i) SPA+LR [22]

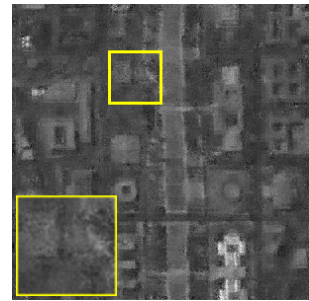

(e) BwK-SVD [17]

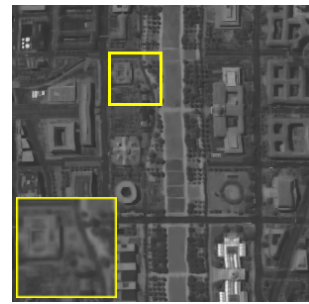

(j) Ours

Fig. 9. (a) The Original image located at the $15^{\text {th }}$ band in the Washington DC Mall dataset; (b) The corrupted image by Gaussian noise of $\sigma=30$; (c)-(f) The denoised hyperspectral images obtained by the comparing denoising methods. Image patches at the bottom-left are zoomed-in views from the top-right small patches.

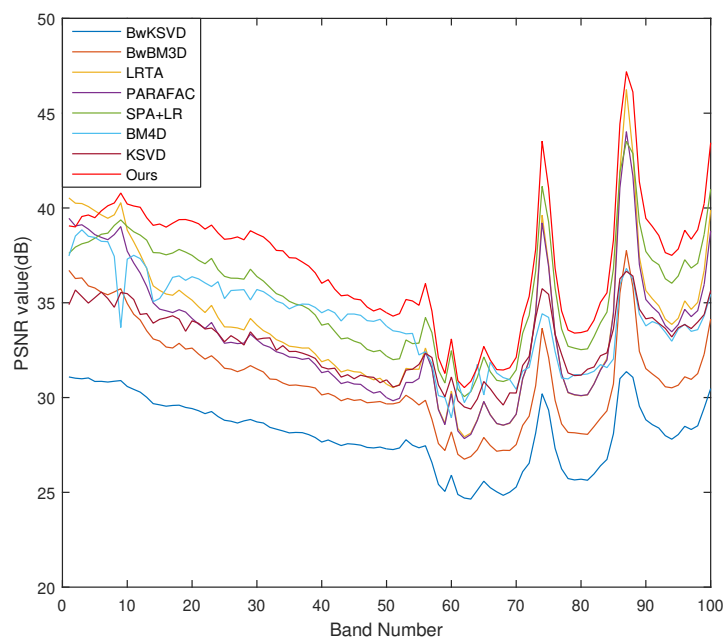

Fig. 10. Comparison of PSNR values among various methods on the Washington DC Dataset with $\sigma=30$.

hyperspectral images," Journal of the Optical Society of America A Optics Image Science and Vision, vol. 34, no. 2, pp. 252, 2017.

[36] S. Meng, L. Huang, and W. Wang, "Tensor decomposition and PCA jointed algorithm for hyperspectral image denoising," IEEE Geoscience and Remote Sensing Letters, vol. 13, no. 7, pp. 897-901, 2016.

[37] W. Cao, Y. Wang, C. Yang, X. Chang, Z. Han, and Z. Xu, "Foldedconcave penalization approaches to tensor completion," Neurocomputing, vol. 152, no. C, pp. 261-273, 2015.

[38] X. Zhao, F. Wang, T. Huang, and M. Ng, "Deblurring and sparse unmixing for hyperspectral images," IEEE Transactions on Geoscience and Remote Sensing, vol. 51, no. 7, pp. 4045-4058, 2013.

[39] R. Kawakami, Y. Matsushita, J. Wright, and M. Ben-Ezra, "Highresolution hyperspectral imaging via matrix factorization," in Computer Vision and Pattern Recognition, 2011, pp. 2329-2336.

[40] M. Ye, Y. Qian, and J. Zhou, "Multitask sparse nonnegative matrix factorization for joint spectral-spatial hyperspectral imagery denoising," IEEE Transactions on Geoscience and Remote Sensing, vol. 53, no. 5, pp. 2621-2639, 2015 .

[41] N. Acito, M. Diani, and G. Corsini, "Signal-dependent noise modeling and model parameter estimation in hyperspectral images," IEEE Transactions on Geoscience and Remote Sensing, vol. 49, no. 8, pp. 2957-2971, 2011.

[42] H. Akaike, "A new look at the statistical model identification," IEEE Transactions on Automatic Control, vol. 19, no. 6, pp. 716-723, 1974.

[43] H. Chen, "Estimating the dimension of a model," Asia-Pacific Journal of Risk and Insurance, vol. 6, no. 2, pp. 461-4, 1978.

[44] A. Cichocki, R. Zdunek, A. Phan, and S. Amari, Nonnegative Matrix and Tensor Factorizations - Applications to Exploratory Multi-way Data Analysis and Blind Source Separation, Wiley, 2009.

[45] A. Phan and A. Cichocki, "Extended HALS algorithm for nonnegative tucker decomposition and its applications for multiway analysis and classification," Neurocomputing, vol. 74, no. 11, pp. 1956-1969, 2011.

[46] F Yasuma, T Mitsunaga, D Iso, and S. K. Nayar, "Generalized assorted pixel camera: postcapture control of resolution, dynamic range, and spectrum.," IEEE Transactions on Image Processing A Publication of the IEEE Signal Processing Society, vol. 19, no. 9, pp. 2241-53, 2010.

[47] [Online], "Hyperspectral remote sensing scenes," http://engineering. purdue.edu/ biehl/MultiSpec/hyperspectral.html.

[48] Y. Tarabalka, J. Benediktsson, and J. Chanussot, "Spectral-spatial classification of hyperspectral imagery based on partitional clustering techniques," IEEE Transactions on Geoscience and Remote Sensing, vol. 47 , no. 8, pp. 2973-2987, 2009. 